\title{
A Review of mathematical models for prediction of Stress- strain and moment -curvature behaviour in concrete
}

\author{
Y Shashank Babu ${ }^{1}$, V Srinivasa Reddy ${ }^{2}, S$ P Raju $\mathrm{V}^{3}, M$ V Seshagiri Rao ${ }^{4}$ \\ ${ }^{1}$ M.Tech (Structural Engineering), Department of Civil Engineering, GRIET, Hyderabad, India. \\ ${ }^{2}$ Professor of Civil Engineering, GRIET, Hyderabad, India. \\ ${ }^{3}$ Assistant Professor of Civil Engineering, GRIET, Hyderabad, India. \\ ${ }^{4}$ Professor of Civil Engineering, CVR College of Engineering, Hyderabad, India.
}

\begin{abstract}
In this paper, a mathematical model for predicting the stress -strain and moment curvature relations in concrete is developed. A good number of empirical equations were proposed to represent stress-strain behaviour of conventional concrete. Most of the equations can be used for the ascending portion of the curve only. In 1997 Mansur et al. have adopted Carriera and Chu (1985) model, which was based on the model proposed by Popovics (1973). As such, model proposed by Mansur et al includes both ascending and descending portions of the stress-strain curve for the confined concrete with introduction of two constants for the descending portion of the curve. Several researchers proposed various empirical equations for stress-strain behaviour as briefly reported in the previous chapter. An attempt has been made in this study to develop mathematical models for concrete in unconfined state. These analytical equations can be applied to any concrete with slight modifications. These models are developed to validate the experimental values obtained.
\end{abstract}

\section{Introduction}

Graph obtained by drawing a curve for the values of stresses and strains obtained during testing a material specimen of materials is called a stress - strain curve. By testing cylinders of standard size made with concrete, under uni-axial compression values of stresses and strains are obtained and the stress-strain curves are plotted. Even though the stress strain relation for cement paste and aggregate when tested individually is practically linear, it is observed from the stress-strain plots of concrete that, no portion of the curves is in the form of a straight line. In concrete the rate of increase of stress is less than that of increase in strain because of the formation of micro cracks, between the interfaces of the aggregate and the cement paste. Thus the stress strain curve is not linear. In conventional concrete the value of stress is maximum corresponding to a strain of about 0.002 and further goes on decreasing with the increasing strain, giving a dropping curve till it terminates at ultimate crushing strain.

\section{Analytical Stress-strain equations}

Number of empirical equations for stress-strain curve has been proposed for conventional concrete. Early works done by Hognestad and Desayi and Krishnan and proposed a basic model for stress-strain of ordinary concrete. Later Saenz has proposed model duly overcoming the limitation in the model of Desayi and Krishnan. Carriera and Chu [7] provided an extension to the empirical equation proposed by Popovics. Further, Loove improves the early work by Carriera and $\mathrm{Chu}$ who proposed a model that can be validate experimental values. Collins et al. also extended the work by Thorenfeldt et al. to examine the relation between compressive stresses at any strain to peak stress. Stressstrain equations proposed by these authors are summarized in the following sections.

\section{Hognestad Model}

Stress-strain relation for ordinary concrete upto ascending portion of stress-strain curve defined by

$\frac{\sigma_{c}}{f_{c}}=\left[\frac{2 \varepsilon}{\varepsilon_{0}}-\left(\frac{\varepsilon}{\varepsilon_{0}}\right)^{2}\right]$

Where

$\sigma_{c}=$ Peak stress in concrete,

$\varepsilon_{0}=$ strain at peak stress,

$f_{c}=$ compressive strength of concrete

$\varepsilon=$ strain in concrete 


\section{Desayi and Krishnan Model}

Desayi and Krishnan proposed simple model describing stress-strain for normal concrete as below

$$
\frac{f_{c}}{f_{c}^{\prime}}=\frac{\left(\frac{\varepsilon}{\varepsilon_{0}}\right) A}{A+B\left(\frac{\varepsilon}{\varepsilon_{0}}\right)^{2}}
$$

Model is derived from Saenz's original equation is in simple form such that closed-form integration can be evaluated to calculate the stress-block parameters. Due to simplicity in model formulation has encouraged many researchers to use it as general stress-strain model for concrete.

\section{Modified saenz model}

Desayi and Krishnan has proposed model for ascending portion of stress-strain curve only. In view of this limitation, Saenz proposed a model considering both ascending and descending portion of stress-strain curve.

\section{Wang et al. Model}

$$
\frac{f_{c}}{f_{c}^{\prime}}=\frac{\left(\frac{\varepsilon}{\varepsilon_{0}}\right) A}{1+B\left(\frac{\varepsilon}{\varepsilon_{0}}\right)+C\left(\frac{\varepsilon}{\varepsilon_{0}}\right)^{2}}
$$

The model used by Wand et al. in the form

$$
\frac{f_{c}}{f_{c}^{\prime}}=\frac{\left(\frac{\varepsilon}{\varepsilon_{0}}\right) A+B\left(\frac{\varepsilon}{\varepsilon_{0}}\right)^{2}}{1+C\left(\frac{\varepsilon}{\varepsilon_{0}}\right)+D\left(\frac{\varepsilon}{\varepsilon_{0}}\right)^{2}}
$$

Carreira and Chu Model

The following general equation represents the stressstrain behaviour proposed by Carreria and Chu

$$
\begin{aligned}
& \qquad \frac{f_{c}}{f_{c}^{\prime}}=\frac{\left(\frac{\varepsilon}{\varepsilon_{0}}\right) \beta}{\beta-1+\left(\frac{\varepsilon}{\varepsilon_{0}}\right)^{\beta}} \\
& \text { Material parameters, } \beta=1 /\left[1-\left(\frac{f_{c}^{\prime}}{E_{0} \varepsilon_{0}}\right)\right] \\
& \text { Where } \\
& \mathrm{V}=\text { Initial tangent modulus }
\end{aligned}
$$

\section{Loove Model}

Loove extended the early work by Carriera and $\mathrm{Chu}$ and proposed as

Where

$$
\frac{f_{c}}{f_{c}^{\prime}}=\frac{\left(\frac{\varepsilon}{\varepsilon_{0}}\right) A}{1+C\left(\frac{\varepsilon}{\varepsilon_{0}^{\prime}}\right)+D\left(\frac{\varepsilon}{\varepsilon_{0}}\right)^{n}}
$$

$f_{c}^{\prime}=$ Cylinder ultimate compressive strength

$\mathrm{A}=1+\mathrm{B}+\mathrm{C}, \mathrm{C}=1 / \mathrm{n}-1$

Indian Standard IS: 456-2000 allows assumption of suitable relationship between the compressive stress distribution and strain as rectangular, trapezoidal, parabola or any other shape which results in prediction of strength followed by substantial agreement with experimental results.

\section{Mathematical model for Concrete}

In the present investigation only ascending portion of curve is considered. Out of existing, Modified Saenz's model is selected which seem to be valid for ascending portion of stress strain curve. The proposed equation of the curve is in the form of

$$
Y=\frac{A X}{1+B X+C X^{2}}
$$

Where $\mathrm{Y}$ is the normalized stress $\left(\frac{\sigma}{\sigma_{u}}\right)$ and $\mathrm{X}$ is the normalized $\operatorname{strain}\left(\frac{\varepsilon}{\varepsilon_{u}}\right)$. A, B, C are the constants. Further, equation for non-dimensional stress-strain curve can be written in the following form

$$
\left(\frac{\sigma}{\sigma_{u}}\right)=\frac{A^{1}\left(\frac{\varepsilon}{\varepsilon_{u}}\right)}{1+B^{1}\left(\frac{\varepsilon}{\varepsilon_{u}}\right)+C^{1}\left(\frac{\varepsilon}{\varepsilon_{u}}\right)^{2}}
$$

The constants $A^{1}, B^{1}, C^{1}$ are determined from the boundary conditions of the non-dimensional stressstrain curve. The boundary conditions are as follows. At the origin, $\sigma=0$ and $\varepsilon=0$, Slope of the stress-strain curve are evaluated using following equations.

$\mathrm{A}=A^{1}\left(\frac{\sigma}{\varepsilon}\right), \mathrm{B}=B^{1}\left(\frac{1}{\varepsilon}\right)$, and $\mathrm{C}=C^{1}\left(\frac{1}{\varepsilon}\right)^{2}$

Finally, substituting the above constants, theoretical equation for the stress-strain curve is obtained for concrete. Theoretical stresses are calculated for corresponding strains using developed equations. These theoretical stresses are compared with experimental results of Normal Concrete.

Boundary conditions for ascending and descending portions of stress-strain curves are,

i. At the origin the ratio of stresses and strains are zero

i.e. at origin $(€ / \epsilon 0)=0,(\sigma / \sigma 0)=0$

$\epsilon 0$ - strain at peak stress, $\sigma 0$ - peak stress

ii. The strain ratio and stress ratio at the peak of the nondimensional stress-strain curve is unity.

i.e. at $(€ / € 0)=1,(\sigma / \sigma 0)=1$

iii. The slope of non-dimensional stress-strain curve at the peak is

zero i.e at $(\epsilon / \epsilon 0)=1.0 \mathrm{~d}(\sigma / \sigma 0)=0$

$\mathrm{d}(\mathrm{C} / \mathrm{C} 0)$

iv. At $85 \%$ stress ratio the corresponding values of strain ratio is 1.3 . I.e at $(\sigma / \sigma 0)=0.85(€ / € 0)=1.3$

Where $\sigma 0$ - corresponds to peak stress and

$\mathrm{C} 0$ - corresponds to strain at peak stress

$\sigma$ and $\epsilon$ corresponds to stress and strain values at any other point

Boundary conditions $i$ and ii are for determining the constants in the ascending portion of the normalized stress-strain curve and ii, iii and iv are for determining the constants in the descending portion of the curve. 
Using the boundary conditions in the non-dimensional stress-strain curves, constants for different SCC mixes are determined and from that the equations are developed.

\section{Stress Block Parameter for Normal Concrete}

Stress-block parameters are essential for the analysis and design of structured members. Using these parameters, flexure capacity of beam can be determined. If assumed stress-strain model is correct, more reliability in strength estimate and deformation behaviour of concrete structural members.

\section{Formulation of stress-block parameters}

As fewer studies on stress-strain behaviour on Normal Concrete are available, therefore development of stressstrain model is necessary to judge the flexural behaviour of Normal Concrete. Modified Saenz's model which was considered for development of stress-strain curves of Normal Concrete. Step by step procedures involved in development of stress-block parameters are illustrated in subsequent sections.

The expressions for compressive force is given by

$$
C_{u}=\propto f_{c u} b X_{u}
$$

The area under stress-strain curve $\left(\mathrm{A}_{b}\right)$ is given by

$\mathrm{A}_{\mathrm{b}}=\propto f_{c u} \varepsilon_{c u}$

Therefore,

$$
\propto=A_{b} / f_{c u} \in_{c u}
$$

Where $\propto$ is average concrete stress ratio without partial safety factor.

Substituting

$C_{u}=\left[b X_{u} / \varepsilon_{c u}\right] A_{b}$

Tensile force $(\mathrm{T})$ is expressed as

$\mathrm{T}=\mathrm{f}_{\mathrm{y}} \times \mathrm{A}_{\mathrm{st}}$

As per Clause 38.1 of IS: $456-2000$

$$
\varepsilon_{s}=\left[0.87 \times \frac{f_{y}}{E_{s}}\right]+0.002
$$

Hence,

Substituting

$$
f_{y}=\frac{E_{s}\left[\varepsilon_{s} \times 0.002\right]}{0.87}
$$

$\mathrm{T}=\mathrm{E}_{\mathrm{s}}\left[\varepsilon_{s}-0.002\right] / 0.87 \times A_{s t}$

$\mathrm{Cu}$ and $\mathrm{T}$ gives the expression for compressive force and tensile force.

\section{Equation for area under stress-strain curve}

Area under stress-strain curve $A_{b}$ is given by

$=\int f d x$

$=\int \frac{A \varepsilon}{1+B \varepsilon+C \varepsilon^{2}} d x$

$=\frac{A}{2 C} \int \frac{2 C \varepsilon+B-B}{1+B \varepsilon+C \varepsilon^{2}} d x$

$=\frac{A}{2 C} \int \frac{B+2 C \varepsilon}{1+B \varepsilon+C \varepsilon^{2}} d x-\frac{A B}{2 C} \int \frac{1}{1+B \varepsilon+C \varepsilon^{2}} d x$

$=\frac{A}{2 C} \int \frac{B+2 C \varepsilon}{1+B \varepsilon+C \varepsilon^{2}} d x-\frac{A B}{2 C^{2}} \int \frac{1}{\frac{1}{C}+\frac{B}{C} \varepsilon+\varepsilon^{2}} d x$

$$
=\frac{A}{2 C} \int \frac{B+2 C \varepsilon}{1+B \varepsilon+C \varepsilon^{2}} d \varepsilon-\frac{A B}{2 C^{2}} \int \frac{1}{\left[\varepsilon+\left(\frac{B}{2 c}\right)\right]^{2}+\frac{4 c-B^{2}}{4 c^{2}}} d \varepsilon
$$

$=\frac{A}{2 C} K_{1}-\frac{A B}{2 C_{2}} K_{2}$

Where

$$
\begin{aligned}
K_{1} & =\log \left(1+B \varepsilon+C \varepsilon^{2}\right) \\
K_{2} & =\int \frac{1}{\frac{1}{C}+\frac{B}{C} \varepsilon+\varepsilon_{2}} d \varepsilon \text { or } K_{2} \\
& =\int \frac{1}{\left[\varepsilon+\left(\frac{B}{2 C}\right)\right]^{2}+\frac{4 C-B^{2}}{4 C^{2}}} d \varepsilon
\end{aligned}
$$

Solution for $K_{2}$ with $\int \frac{1}{\frac{1}{C}+\frac{B}{C} \varepsilon+\varepsilon_{2}} d \varepsilon$ :-

It is in the form of $\int \frac{A x+D}{q+p x+x^{2}} d \varepsilon$ i.e., denominator is quadratic irreducible with numerator is constant or linear.

The three solutions for ' $\mathrm{k}_{2}$ ' depending up on discriminant i.e., $p^{2}-4 q$

Case I :- If $p^{2}-4 q$ is $+v e$

$=\frac{A \propto 1+D}{\alpha 1-\alpha 2} \ln \left[\varepsilon-\alpha_{1}\right]+\frac{A \alpha_{2}+D}{\alpha_{2}-\alpha_{1}} \ln \left[\varepsilon-\alpha_{2}\right]$

$\alpha_{1}=\frac{-p-\sqrt{p^{2}-4 q}}{2}, \alpha_{2}=\frac{-p+\sqrt{p^{2}-4 q}}{2}$

Substituting

$\mathrm{P}=\frac{B}{C}, \mathrm{q}=\frac{1}{C}$

$\alpha_{1}=\frac{-\frac{B}{C}-\sqrt{\left(\frac{B}{C}\right)^{2}-4 \frac{1}{C}}}{2}$

$=\frac{-B-\sqrt{B^{2}-4 C}}{2 C}$, Let $\mathrm{Q}-\sqrt{B^{2}-4 C}$

$=\frac{-B-Q}{2 c}$

$\alpha_{1}=-\frac{(B+Q)}{2 c}$,

Similarly $\alpha_{2}=-\frac{(B-Q)}{2 c}$

$\alpha_{1}-\alpha_{2}=-\frac{(B+Q)}{2 c}-\left[-\frac{(B-Q)}{2 c}\right]=-\frac{Q}{C}$

$\alpha_{1}-\alpha_{2}=-\frac{(B-Q)}{2 c}-\left[-\frac{(B+Q)}{2 c}\right]=\frac{Q}{C}$

$\varepsilon-\alpha_{1}=\varepsilon-\left[-\frac{(B+Q)}{2 c}\right]=\frac{2 C \varepsilon+B+Q}{2 C}$

Similarly $\varepsilon-\alpha_{2}=\frac{2 C \varepsilon+B-Q}{2 C}$

Substituting above values and also $\mathrm{A}=0, \mathrm{D}=1$

$=\frac{1}{\frac{Q}{C}} \ln \left[\frac{2 C \varepsilon+B+Q}{2 C}\right]+\frac{1}{\frac{Q}{C}} \ln \left[\frac{2 C \varepsilon+B-Q}{2 c}\right]$

$=-\frac{C}{Q} \ln [2 c+B+Q]+\frac{1}{2 Q} \ln [2 c+B-Q]$

$K_{2}=\frac{C}{Q} \log \frac{(2 c \varepsilon+B-Q)}{(2 c \varepsilon+B+Q)}$

Case II :- If $\mathrm{p}^{2}-4 \mathrm{q}$ is zero

$=\mathrm{A} \ln [\varepsilon-\sigma]-\frac{A \sigma+D}{\varepsilon-\sigma}$

Substituting, $\sigma=-\frac{p}{2}=-\frac{B}{2 C}$, and $A=0, D=1$ 


$$
\begin{aligned}
& =-\frac{1}{\varepsilon-\left(-\frac{B}{2 C}\right)} \\
& K_{2}=-\frac{2 C}{2 C \varepsilon+B}
\end{aligned}
$$

Case III :- If p2- $4 \mathrm{q}-\mathrm{ve}$

Where,

$$
=\frac{A}{2} \ln \left[(\varepsilon-\beta)^{2}+\gamma^{2}\right]+\frac{A \beta+D}{\gamma} \tan ^{-1}\left(\frac{\varepsilon-\beta}{\gamma}\right)
$$

$\beta=-\frac{p}{2}=-\frac{B}{2 c}$,

$\gamma=\sqrt{q-\left(\frac{p^{2}}{4}\right)}=\sqrt{\frac{1}{c}-\frac{B^{2}}{4 C^{2}}}=\sqrt{\frac{4 C-B^{2}}{2 C}}$

Substituting $\beta, \gamma$ and $A=0, D=1$

$=\frac{1}{\gamma} \tan ^{-1}\left(\frac{\varepsilon-\beta}{\gamma}\right)$

$=\frac{1}{\sqrt{\frac{4 C-B^{2}}{2 C}}} \tan ^{-1}\left(\frac{\varepsilon-\left(-\frac{B}{2 C}\right)}{\sqrt{\frac{4 C-B^{2}}{2 C}}}\right)$

$=\frac{2 C}{\sqrt{4 C-B^{2}}} \tan ^{-1}\left(\frac{\left(\frac{2 C \in+B}{2 C}\right)}{\sqrt{\frac{4 C-B^{2}}{2 C}}}\right)$

$\mathrm{K}_{2}=\frac{2 C}{\sqrt{4 C-B^{2}}} \tan ^{-1}\left(\frac{\left(\frac{2 C \in+B}{2 C}\right)}{\sqrt{\frac{4 C-B^{2}}{2 C}}}\right)$

Solution for $\mathrm{K}_{2}$ With $\int \frac{1}{\left[\varepsilon+\left(\frac{B}{2 C}\right)^{2}+\frac{4 C-B^{2}}{4 C^{2}}\right.} d \varepsilon$ :-

The three solutions for $\mathrm{k}_{2}$ depending up on " $4 \mathrm{C}-\mathrm{B}^{2}$ ",

Case I :- If $4 \mathrm{C}-\mathrm{B}^{2}$ is $-\mathrm{ve}$ or $\left(\mathrm{B}^{2}-4 \mathrm{c}\right.$ is $\left.+\mathrm{ve}\right)$

Let $4 \mathrm{c}-\mathrm{B}^{2}=-\mathrm{Q}^{2}$

$=\int \frac{1}{\left[\varepsilon+\left(\frac{B}{2 C}\right)\right]^{2}-\frac{Q^{2}}{4 c^{2}}} d \varepsilon$

$=\int \frac{1}{\left[\varepsilon+\left(\frac{B}{2 C}\right)\right]^{2}-\left(\frac{Q}{2 C}\right)^{2}} d \varepsilon$

It is in the standard form, $\int \frac{1}{x^{2}-a^{2}} \mathrm{dX}=\frac{1}{2 a} \ln \frac{(X-a)}{(X+a)}$

Substitute for

$\mathrm{X}=\varepsilon+\left(\frac{B}{2 C}\right) \& a=\left(\frac{Q}{2 C}\right)$

$=\frac{1}{2\left(\frac{Q}{2 C}\right)} \ln \frac{\left(\varepsilon+\left(\frac{B}{2 C}\right)-\left(\frac{Q}{2 C}\right)\right)}{\left(\varepsilon+\left(\frac{B}{2 C}\right)+\left(\frac{Q}{2 C}\right)\right)}$

$\mathrm{K}_{2}=\frac{C}{Q} \ln \frac{(2 c \varepsilon+B-Q)}{(2 c \varepsilon+B+Q)}$

Case II :- If $4 \mathrm{c}-B^{2}$ is Zero

Let $4 \mathrm{c}-B^{2}=0$

$=\int \frac{1}{\left[\varepsilon+\left(\frac{B}{2 C}\right)\right]^{2}} d \varepsilon$

$=4 c^{2} \int \frac{1}{[2 c \varepsilon+B]^{2}} d \varepsilon$

Let $\mathrm{U}=2 \mathrm{c} \varepsilon+B$

$\frac{d u}{d \varepsilon}=2 C$ or $\frac{d u}{2 c}=d \varepsilon$

$=4 c^{2} \int \frac{1}{[U]^{2}} \frac{d u}{2 c}$

$=2 \mathrm{c} \int \frac{1}{[U]^{2}} d u$

$=2 \mathrm{c}\left[\frac{U^{-2+1}}{-2+1}\right]$
$=2 \mathrm{c}\left[\frac{U^{-1}}{-1}\right]$

$=-2 \mathrm{c}\left[\frac{1}{-U}\right]$

$\mathrm{K}_{2}=-\frac{2 C}{2 C \epsilon+B}$

Case III :- If $4 \mathrm{c}-B^{2}$ is + ve

Let $4 c-B^{2}=R^{2}$

$=\int \frac{1}{\left[\varepsilon+\left(\frac{B}{2 C}\right)\right]^{2}+\frac{R^{2}}{4 C^{2}}} d \epsilon$

$=\int \frac{1}{\left[\varepsilon+\left(\frac{B}{2 C}\right)\right]^{2}+\left(\frac{R}{2 C}\right)^{2}} d \epsilon$

It is in the standard form $\int \frac{1}{x^{2}+a^{2}} d x=\frac{1}{a} \tan ^{-1} \frac{x}{a}$

Substitute for

$\mathrm{X}=\varepsilon+\left(\frac{B}{2 c}\right) \& a=\left(\frac{R}{2 C}\right)$

$=\frac{1}{\left(\frac{R}{2 C}\right)} \tan ^{-1}\left(\frac{\epsilon+\left(\frac{B}{2 C}\right)}{\left(\frac{R}{2 C}\right)}\right)$

$=\frac{2 C}{R} \tan ^{-1}\left(\frac{2 c \epsilon+B}{R}\right)$

Substitute $\sqrt{4 c-B^{2}}=R$

$\mathrm{K}_{2}=\frac{2 c}{\sqrt{4 c-B^{2}}} \tan ^{-1}\left(\frac{2 c \epsilon+B}{\sqrt{4 C-B^{2}}}\right)$

\section{Equation for centroid distance of area stress-} strain from neutral axis $\mathbf{N}_{\mathbf{x}}$

$\mathrm{N}_{\mathrm{x}}=\frac{\int f \cdot \varepsilon d \varepsilon}{\int f d \varepsilon}$

$=\frac{1}{A s} \int f \varepsilon d \varepsilon$

$\int f \varepsilon d \epsilon=\int \frac{A \varepsilon}{1+B \epsilon+C \varepsilon^{2}} \varepsilon d \varepsilon$

$=\mathrm{A} \int \frac{\varepsilon 2}{1+B \epsilon+C \varepsilon^{2}} d \varepsilon=\frac{A}{C} \int \frac{C \varepsilon 2}{1+B \epsilon+C \varepsilon^{2}} d \varepsilon$

$=\frac{A}{C} \int \frac{1+B \varepsilon+C \varepsilon^{2}-(1+B \varepsilon)}{1+B \varepsilon+C \varepsilon^{2}} d \varepsilon$

$=\frac{A}{C}\left[\int \frac{1+B \varepsilon+C \varepsilon^{2}}{1+B \varepsilon+C \varepsilon^{2}} d \varepsilon-\int \frac{(1+B \varepsilon)}{1+B \varepsilon+C \varepsilon^{2}} d \varepsilon\right]$

$=\frac{A}{C}\left[\int d \varepsilon-\int \frac{1}{1+B \varepsilon+C \varepsilon^{2}} d \varepsilon-\int \frac{B \epsilon}{1+B \varepsilon+C \varepsilon^{2}} d \varepsilon\right]$

$=\frac{A}{C}\left[\int d \varepsilon-\int \frac{1}{1+B \varepsilon+C \varepsilon^{2}} d \varepsilon-\frac{B}{2 C} \int \frac{2 C \epsilon}{1+B \varepsilon+C \varepsilon^{2}} d \varepsilon\right]$

$=\frac{A}{C}\left[\int d \varepsilon-\int \frac{1}{1+B \varepsilon+C \varepsilon^{2}} d \varepsilon-\frac{B}{2 C} \int \frac{2 C \epsilon+B-B}{1+B \varepsilon+C \varepsilon^{2}} d \varepsilon\right]$

$=\frac{A}{C}\left[\int d \varepsilon-\int \frac{1}{1+B \varepsilon+C \varepsilon^{2}} d \varepsilon-\frac{B}{2 C}\left\{\int \frac{2 C \epsilon+B}{1+B \varepsilon+C \varepsilon^{2}} d \varepsilon\right]\right.$

$\left.\left.\int \frac{B}{1+B \varepsilon+C \varepsilon^{2}} d \varepsilon\right\}\right]$

$=\frac{A}{C}\left[\int d \varepsilon-\int \frac{1}{1+B \varepsilon+C \varepsilon^{2}} d \varepsilon-\frac{B}{2 C}\left\{\int \frac{2 C \epsilon+B}{1+B \varepsilon+C \varepsilon^{2}} d \varepsilon\right]\right.$

$\left.\left.+\frac{B^{2}}{2 C} \int \frac{B}{1+B \varepsilon+C \varepsilon^{2}} d \varepsilon\right\}\right]$

$\left.=\frac{A}{C}\left[\int d \varepsilon-\frac{B}{2 C} \int \frac{2 C \epsilon+B}{1+B \varepsilon+C \varepsilon^{2}} d \varepsilon\right]+\left(\frac{B^{2}}{2 C}-1\right) \int \frac{B}{1+B \varepsilon+C \varepsilon^{2}} d \varepsilon\right]$

$\left.=\frac{A}{C}\left[\int d \varepsilon-\frac{B}{2 C} \int \frac{2 C \epsilon+B}{1+B \varepsilon+C \varepsilon^{2}} d \varepsilon\right]+\left(\frac{B^{2}}{2 C}-1\right) \frac{1}{C} \int \frac{B}{1+B \varepsilon+C \varepsilon^{2}} d \varepsilon\right]$ on Integration ,

$=\frac{A}{C}\left[\varepsilon-\frac{B}{2 C} K_{1}+\frac{1}{C}\left(\frac{B^{2}}{2 C}-1\right) K_{2}\right.$

$=\frac{A}{C}\left[\varepsilon-\frac{B}{2 C} K_{1}+\frac{1}{2 C^{2}}\left(B^{2}-2 c\right) K_{2}\right.$

$=\frac{A}{2 C^{3}}\left[2 C^{2} \epsilon-B C K_{1}+\left(B^{2}-2 \mathrm{c}\right) K_{2}\right.$

Therefore 
$\int f . \epsilon d \epsilon=\frac{A}{2 C^{3}}\left[2 C^{2} \varepsilon-B C K_{1}+\left(B^{2}-2 C\right) K_{2}\right]$

Substituting

$A_{b}=\frac{A}{2 C} K_{1}-\frac{A B}{2 C_{2}} K_{2}$

$\int f \varepsilon d \varepsilon=\frac{A}{2 c^{3}}\left[2 C^{2} \varepsilon-B C K_{1}+\left(B^{2}-2 C\right) K_{2}\right]$

In $\frac{1}{A s} \int f \varepsilon d \varepsilon$

Substituting above,

$=\frac{\frac{A}{2 c^{3}}\left(2 c^{2} \epsilon-B C K_{1}+\left(B^{2}-2 C\right) K_{2}\right.}{\frac{A}{2 C^{2}}\left(C K_{1}-B K_{2}\right)}$

$=\frac{\left(2 c^{2} \epsilon-B C K_{1}+\left(B^{2}-2 C\right) K_{2}\right.}{C\left(C K_{1}-B K_{2}\right)}$

Thus, centroid distance of area from neutral axis

$N_{x} \frac{\int f \varepsilon d \varepsilon}{\int f d \varepsilon}=\frac{\left(2 c^{2} \varepsilon-B c K_{1}+\left(B^{2}-2 C\right) K_{2}\right.}{C\left(C K_{1}-B K_{2}\right)}$

Hence, expression for compressive force $\left(c_{u}\right)$ is given by equation

$\left[\mathrm{b} X_{U} / \varepsilon_{c u}\right]\left(\frac{A}{2 C} K_{1}-\frac{A B}{2 C_{2}} K_{2}\right)$

Where

$\mathrm{C}_{\mathrm{u}}$ is compressive force

$\mathrm{b}$ is breadth of the section

$X_{u}$ is depth of neutral axis

$\varepsilon_{c u}$ is the ultimate strain in concrete

$\mathrm{A}, \mathrm{B} \& \mathrm{C}$ are the constants obtained from stress-strain curves

\section{Calculation of stress block parameters}

Assume initial trial value $X_{u} / \mathrm{d}$

Using strain-compatibility method determine the $\varepsilon_{s}$ by following equation

$$
\varepsilon_{s}=\epsilon_{c u}\left(\frac{d}{X_{u}}-1\right)
$$

Determine the design stress $\mathrm{f}_{\mathrm{y}}$ corresponding to $\varepsilon_{s}$ using the design stress-strain curve of steel

Determine the value $\mathrm{T}=f_{y} * \mathrm{~A}_{\mathrm{st}}$

The area $\mathrm{AB}$ under stress-strain curve was calculated from equation by taking $\mathrm{f}_{\mathrm{u}}=\mathrm{f}_{\mathrm{cu}}$

The stress block parameter $\propto$ is obtained from equation by taking $\mathrm{f}_{\mathrm{u}}=\mathrm{f}_{\mathrm{cu}}$

The depth of the parabolic portion of the stress-block $\left(\mathrm{x}_{2}\right)$ was obtained from stress-strain diagram and is given by $\mathrm{X}_{1}=\left[\epsilon_{c} / \epsilon_{c u}\right] * X_{U}$

Similarly, the depth of rectangular portion of stressblock $\left(\mathrm{x}_{2}\right)$ is given by

$\mathrm{x}_{2}=\left[\epsilon_{c u}-\epsilon_{c} / \epsilon_{c u}\right] * X_{U}$

Compressive force component for the parabolic portion $\left(\mathrm{c}_{1}\right)$ of stress-block is given by

$\mathrm{c}_{1}=2 / 3 \mathrm{X}_{1}\left(\alpha f_{c u}\right) \mathrm{b}$

given by

$\mathrm{c}_{2}=\mathrm{X}_{2}\left(\alpha f_{c u}\right) \mathrm{b}$

thus total compressive force $\left(c_{u}\right)$ is given by $c_{u}=c_{1}+c_{2}$
Let $\mathrm{X}$ be the distance of the line of the action of compressive force from extreme top fiber, then

$\mathrm{X}=\left[\mathrm{C}_{1}\left(3 / 8 \mathrm{X}_{1}+\mathrm{X}_{2}\right)+\mathrm{C}_{2}\left(\mathrm{X}_{2} / 2\right)\right] / \mathrm{C}_{\mathrm{U}}$

Let $\mathrm{X}=\beta \mathrm{X}_{\mathrm{u}}$

Where $\beta$ is the effective stress-block depth factor and is a function of $X_{1}, X_{2}, C_{1}, C_{2}$ and $C_{U}$ by virtue of the equilibrium, compressive force must equal to tensile force.compare the difference between compressive force and tensile force, if the value is insignificant then calculate $\mathrm{X}_{\mathrm{U}} / \mathrm{d}$ with initially assumed value .If both values are equal stress - block parameters $\alpha$ and $\beta$ Are accepted. Otherwise, repeat the procedure assuming new value of $\mathrm{X}_{\mathrm{U}} / \mathrm{d}$.

\section{Theoretical moments and curvatures}

The experimental results of moments have been analyzed by developing procedures for obtaining the complete theoretical moment-curvature diagrams. The models proposed for stress-strain behaviour of concrete mixes are used as the basis for prediction of the analytical behaviour of moment-curvature and in deriving the expressions of the resisting moments and curvatures. For obtaining the complete moment curvature relationship for any cross-section, discrete values of concrete strains $(E)$ were selected such that even distribution of points on the plot, both before and after maximum was obtained.

The procedure used in the computation is given below.

i) Calculation of neutral axis depth for given values of concrete strains $(\mathcal{C})$

ii) Calculation of moment carrying capacities (M)

iii) Calculation of theoretical moment curvature values

Moment of resistance is given by

$\mathrm{M}_{\mathrm{t}}=\mathrm{C}_{\mathrm{U}} * \mathrm{Z}$

Substituting $\mathrm{f}_{\mathrm{cu}}=\mathrm{f}_{\mathrm{ck}}$

$\mathrm{M}_{\mathrm{t}}=\alpha \mathrm{f}_{\mathrm{ck}} \mathrm{bX}_{\mathrm{u}}\left(\mathrm{d}-\beta \mathrm{X}_{\mathrm{U}}\right)$

Also moment of resistance $\mathrm{M}_{\mathrm{t}}$ is given by $\mathrm{M}_{\mathrm{t}}=\mathrm{k}_{\mathrm{t}} \mathrm{f}_{\mathrm{ck}} \mathrm{bd}^{2}$

$$
\mathrm{K}_{\mathrm{t}}=\mathrm{M}_{\mathrm{t}} / \mathrm{bd}^{2}
$$

Where $\mathrm{k}_{\mathrm{t}}$ is moment resistance factor

$\mathrm{k}_{\mathrm{t}}=\alpha^{\prime} \mathrm{X}_{\mathrm{u}} / \mathrm{d}\left(1-\beta \mathrm{X}_{\mathrm{U}} / \mathrm{d}\right)$

using partial safety factor 1.5

$\mathrm{k}_{\mathrm{t}}=\alpha \mathrm{X}_{\mathrm{u}} / \mathrm{d}\left(1-\beta \mathrm{X}_{\mathrm{U}} / \mathrm{d}\right)$

Theoretical moment is obtained by $\mathrm{M}_{\mathrm{t}}=\mathrm{k}_{\mathrm{t}} \mathrm{f}_{\mathrm{ck}} \mathrm{bd}^{2}$

The resistance factor $K_{t}$ for each grade calculated using developed stress-block parameters

As mentioned earlier, a final phase of experimental was undertaken to validate the stress block and design parameters which are developed.

\section{Conclusions}

After determining the stress-strain behaviour of concrete, empirical equations were developed based on the relevant simplified models proposed by (1) Derived 
Saenz model based on Madrid parabola as adopted by the European Concrete Committee, (2) Modified Saenz model (1964), and (3) model of Mansur et al. (1997) to present uni-axial stress-strain behaviour of concrete mixes and these models were compared with experimental stress-strain behaviour.

\section{Acknowledgements}

We sincerely acknowledge that the most of the information is gathered from research works of my teachers Dr. M V Seshagiri Rao, Retd. Professor of Civil Engineering JNTUH Hyderabad, India and Dr. V Bhikshma, Professor of Osmania University, Hyderabad, India

\section{References}

1. Tasnimi, A. A. "Mathematical model for complete stress-strain curve prediction of normal, lightweight and high-strength concretes." Magazine of concrete research 56.1 (2004): 23-34.

2. Ganesan, N., et al. "Stress-strain behaviour of confined Geopolymer concrete." Construction and building materials 73 (2014): 326-331.

3. Bhikshma. V, and Kishore. R "Development of Stress-Strain Curves for Recycled Aggregate Concrete", Asian Journal of Civil Engineering (Building and Housing) Vol.11, No:2(2010),pp253261.

4. Daniel Cusson and Patrick Paultre, Member, ASCE "Stress- Strain Model for Confined High-Strength Concrete", Journal of Structural Engineering, Vol.121, No.3, March 1995, Paper No. 7257, pp468-477.

5. Domingo. J. Carreira and Kuang-Han Chu, "StressStrain Relationship for Plain Concrete in Compression" ACI Journal Technical Paper Title no. 82-72 November-December1985 pp797-804.

6. Tummala Suresh Kumar, Kosaraju Satyanarayana, Materials Today: Proceeding, 26 (2), 3228-3233, (2020).

7. Insang Lee "Complete Stress-Strain Characteristics of High Performance Concrete" Ph.D Thesis, New Jersey Institute of Technology, May 2002

8. Luis P. Saenz et al "Discussion of the PaperEquation for the Sress-Strain Curve of Concrete by Prakash Desayi et al" ACI journal, proceedings V.61, No.9, September 1964 pp1227-1239 